\title{
Many faces of the Landau gauge gluon propagator at zero and finite temperature: positivity violation, spectral density and mass scales
}

\author{
Paulo J. Silva*, Orlando Oliveira \\ Center for Computational Physics, University of Coimbra \\ E-mail: psilva@teor.fis.uc.pt, orlando@teor.fis.uc.pt
}

\section{David Dudal}

Ghent University, Department of Physics and Astronomy, Krijgslaan 281-S9, 9000 Gent,

\section{Belgium}

E-mail: david.dudal@ugent.be

\section{Pedro Bicudo, Nuno Cardoso ${ }^{\dagger}$}

CFTP, Instituto Superior Técnico, CFTP, Universidade de Lisboa, 1049-001, Lisboa, Portugal E-mail: bicudo@tecnico.ulisboa.pt, nunocardoso@cftp.ist.utl.pt

\begin{abstract}
We address several aspects of gluon propagation at zero and finite temperature. In particular, we study the violation of spectral positivity, we discuss a method to extract the Källén-Lehmann spectral density of a particle (be it elementary or bound state) propagator and apply it to compute gluon spectral densities from lattice data. Furthermore, we also consider the interpretation of the Landau gauge gluon propagator at finite temperature as a massive type bosonic propagator.
\end{abstract}

QCD-TNT-III-From quarks and gluons to hadronic matter: A bridge too far?, 2-6 September, 2013

European Centre for Theoretical Studies in Nuclear Physics and R elated Areas (ECT*), Villazzano, Trento (Italy)

\footnotetext{
*Speaker.

${ }^{\dagger}$ Address after September 2013: NCSA, University of Illinois, Urbana IL 61801, USA.
} 


\section{Gluons at zero temperature}

In recent years, the Landau gauge gluon propagator

$$
D_{\mu v}^{a b}(\hat{p})=\delta^{a b}\left(\delta_{\mu v}-\frac{p_{\mu} p_{v}}{p^{2}}\right) D\left(p^{2}\right)
$$

has been computed on the lattice, using volumes as large as $(27 \mathrm{fm})^{4}$ for the $\mathrm{SU}(2)$ gauge group [1] and $(17 \mathrm{fm})^{4}$ for the SU(3) gauge group [2]. This was due to a renewed interest in the infrared behaviour of the Landau gauge Yang-Mills propagators, in connection with the gluon confinement phenomenon. Simulations show that the propagators reach a finite non-zero value in the infrared region. However, the lattice spacing used in the referred simulations was quite big, being $0.22 \mathrm{fm}$ for $\mathrm{SU}(2)$ and $0.18 \mathrm{fm}$ for SU(3). Despite the large physical volume, the use of such large lattice spacings changes quantitatively the propagator in the infrared region [3]. Although we will not discuss this effect here, it is an important bias, together with the Gribov copies effect $[4,5]$, that should not be forgotten. We call the reader's attention that, in what concerns the ghost propagator, the combined effect of lattice spacing and physical volume was not investigated so far for the SU(3) gauge group ${ }^{1}$.

\subsection{Positivity violation of the gluon propagator as a sign of gluon confinement}

It is a well accepted fact that the $\mathscr{S}$-matrix of a non-Abelian gauge theory does not display poles that would correspond to asymptotically observable degrees of freedom with the quantum numbers of gluons (color charged vector particles). This is a simple empirical fact in the case of QCD: we observe no free quarks or gluons, but we do observe pions, mesons etc.

The strong coupling makes it difficult to address with continuum tools the issue of the nonperturbatively realized QCD spectrum. Useful input can come from gauge fixed lattice simulations of e.g. the quark and gluon propagator. In this proceeding, we will solely focus on pure glue dynamics and ensuing (Euclidean) gluon propagation. From state-of-the-art lattice simulations [7, 8, 9, 10] in the Landau gauge, a numerical estimate can be obtained for the so-called Schwinger function:

$$
C(t)=\int_{-\infty}^{\infty} \frac{\mathrm{d} p}{2 \pi} D\left(p^{2}\right) \exp (-i p t) .
$$

With some complex analysis tools, one can then link $C(t)$ to the Källén-Lehmann spectral function $\rho\left(\omega^{2}\right)$ of the gluon:

$$
C(t)=\int_{0}^{\infty} \mathrm{d} \omega \rho\left(\omega^{2}\right) e^{-\omega t},
$$

under the assumption the gluon has a standard Källén-Lehmann representation of the form

$$
D\left(p^{2}\right)=\int_{0}^{\infty} \mathrm{d} \mu \frac{\rho(\mu)}{\mu+p^{2}} .
$$

As $\rho(\mu)$ has the meaning of a scattering probability, it ought to be positive in a physical Hilbert space. From the correspondence (1.3), it is then clear that $C(t)$ should be, at least, also positive.

The gluon Schwinger function $C(t)$ is depicted in Figure 1, clearly displaying a violation of positivity, thence the gluon cannot be attributed a physical meaning. This can be seen as evidence in favour of gluon confinement, see also [11] for more detailed spectral musings.

\footnotetext{
${ }^{1}$ For a SU(2) analysis see [6].
} 


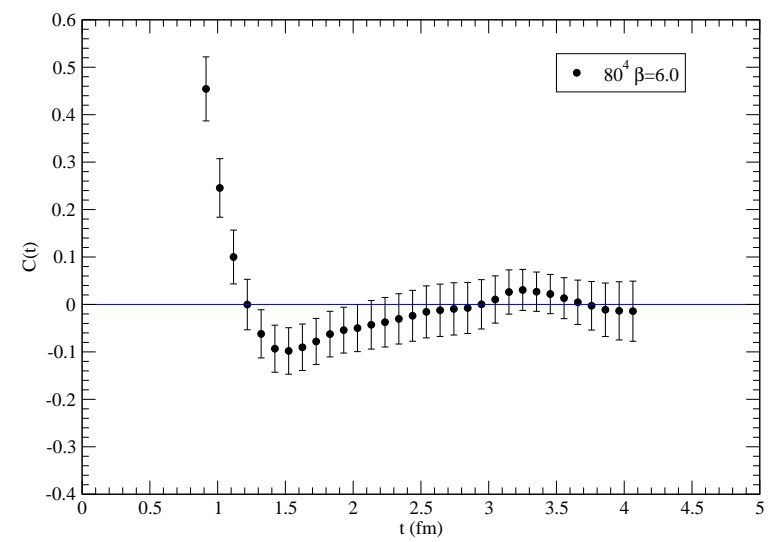

Figure 1: Temporal correlator for the gluon propagator computed using $80^{4} \beta=6.0$ lattice data.

\subsection{Determination of the gluon spectral density from lattice data}

The spectral density contains, amongst other things, information on the masses of physical states described by the operator $\mathscr{O}$.

We now wish, given data input (with errors) for the propagator at a set of discrete momenta, to obtain a stable estimate for the spectral function. In general, this is a inversion problem. It is interesting to notice that eq. (1.4) is equivalent to applying the Laplace transform twice, $D=$ $\mathscr{L}^{2} \hat{\rho}=\mathscr{L} \mathscr{L}^{*} \hat{\rho}$ where $(\mathscr{L} f)(t) \equiv \int_{0}^{\infty} \mathrm{d} s e^{-s t} f(s)$. This is a notorious ill-posed problem. We used $\mathscr{L}=\mathscr{L}^{*}$.

For positive spectral functions, the inversion can be achieved using the maximum entropy method (MEM) [12]. Though, as the gluon Schwinger function already reveals the spectral density cannot be positive over its whole domain, the standard MEM procedure does not apply. We will rely on an alternative approach, preliminary discussed in $[13,14,15]$ with a more complete treatment in [16]. We found inspiration in the Tikhonov approach to ill-posed problems, supplemented with the Morozov discrepancy principle. Specifically, setting $D_{i} \equiv D\left(p_{i}^{2}\right)$ and assuming we have $N$ data points, we minimized

$$
\mathscr{J}_{\lambda}=\sum_{i=1}^{N}\left[\int_{\mu_{0}}^{+\infty} \mathrm{d} \mu \frac{\rho(\mu)}{p_{i}^{2}+\mu}-D_{i}\right]^{2}+\lambda \int_{\mu_{0}}^{+\infty} \mathrm{d} \mu \rho^{2}(\mu)
$$

where we use lattice data in momentum space for the gluon propagator computed in a $80^{4}$ volume, with $\beta=6.0[3,16]$. The data was renormalized in a MOM scheme at $\mu=4 \mathrm{GeV}$ [3]. For $\lambda=0$, we would be searching that $\rho$ that reproduces the data as close as possible in norm. Though, we need $\lambda>0$ as a "screening filter" to overcome the ill-posed nature of the inversion. This amounts to Tikhonov regularization in a discrete setting. The Morozov principle amounts to fix the a priori free parameter $\lambda$ on that value $\bar{\lambda}$ whereby the quality of the inversion is equal to the error on the data, i.e. $\left\|D^{\text {reconstructed }}-D^{\text {data }}\right\|=\delta$ where $\delta$ is the total noise on the input data. We also introduced an IR regulator (threshold) $\mu_{0}$ into the game, the value thereof will be determined self-consistently by means of the optimal (Morozov) regulator $\bar{\lambda}$ : we took the minimal value for $\bar{\lambda}\left(\mu_{0}\right)$ that can be 


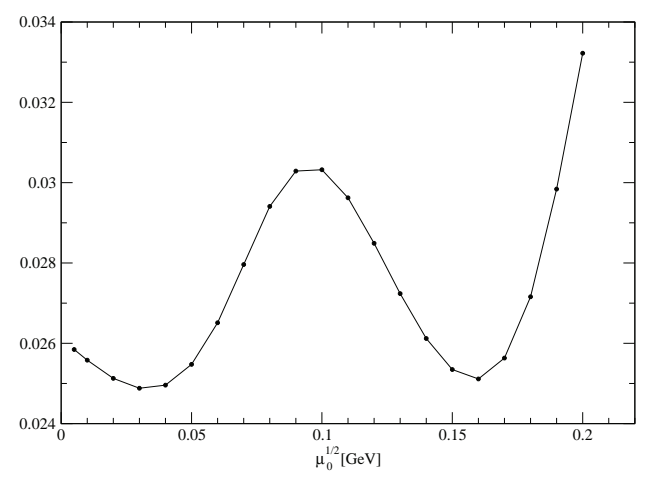

Figure 2: The Morozov parameter $\bar{\lambda}$ in terms of the threshold $\mu_{0}$.

reached by varying $\mu_{0}$. This sounds natural: the smaller $\lambda>0$ becomes the better we approach the original (ill-posed) problem.

Perturbing $\rho(\mu)$ linearly and demanding that the variation of $\mathscr{J}_{\lambda}$ vanishes, leads, after some manipulation, to the following equation that we need to solve for $\rho(\mu)$.

$$
\sum_{i=1}^{N} \underbrace{\left[\int_{\mu_{0}}^{+\infty} \mathrm{d} v \frac{\rho(v)}{p_{i}^{2}+v}-D_{i}\right]}_{\equiv c_{i}} \frac{1}{p_{i}^{2}+\mu}+\lambda \rho(\mu)=0\left(\mu \geq \mu_{0}\right)
$$

Said otherwise, the (regularized) Källén-Lehmann inverse is explicitly given by

$$
\rho_{\lambda}(\mu)=-\frac{1}{\lambda} \sum_{i=1}^{N} \frac{c_{i}}{p_{i}^{2}+\mu} \theta\left(\mu-\mu_{0}\right)
$$

with $\theta(\cdot)$ the Heaviside step function. Combination of eqns. (1.6) \& (1.7) yields a linear system to be solved for the coefficients $c_{i}$ :

$$
\lambda^{-1} \mathscr{M} c+c=-D
$$

with

$$
\mathscr{M}_{i j}=\int_{\mu_{0}}^{+\infty} \mathrm{d} v \frac{1}{p_{i}^{2}+v} \frac{1}{p_{j}^{2}+v}=\frac{\ln \frac{p_{j}^{2}+\mu_{0}}{p_{i}^{2}+\mu_{0}}}{p_{j}^{2}-p_{i}^{2}} .
$$

The reconstructed propagator, which depends on $\lambda$, can be directly expressed as follows:

$$
D^{\text {reconstructed }}\left(p^{2}\right)=\int_{\mu_{0}}^{+\infty} \mathrm{d} \mu \frac{\rho_{\lambda}(\mu)}{p^{2}+\mu}=-\frac{1}{\lambda} \sum_{i=1}^{N} \frac{c_{i} \ln \frac{p^{2}+\mu_{0}}{p_{i}^{2}+\mu_{0}}}{p^{2}-p_{i}^{2}} .
$$

The highest accessible lattice momenta reads $p_{\max }=7.77 \mathrm{GeV}$, a value that we will use here as an UV cut-off. The number of lattice data points was 124 and the noise level comes as $\delta=$ $0.658 \mathrm{GeV}^{-2}$.

In Figure 2 we notice the occurrence of 2 minima for $\bar{\lambda}\left(\mu_{0}\right)$, at $\mu_{0} \approx 0.03 \mathrm{GeV}^{2}$ and $\mu_{0} \approx$ $0.16 \mathrm{GeV}^{2}$, with the former one giving a slightly lower value of $\bar{\lambda}$. For both values, the reconstructed propagator and associated spectral density are shown in Figure 3. We clearly observe that 


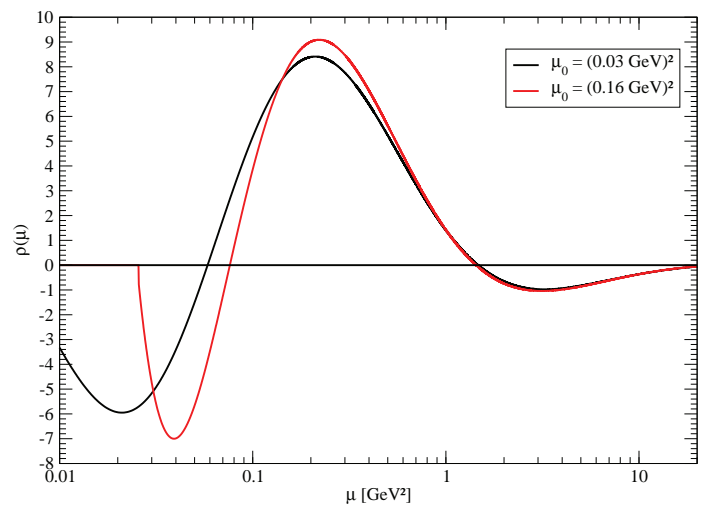

(a) Spectral density.

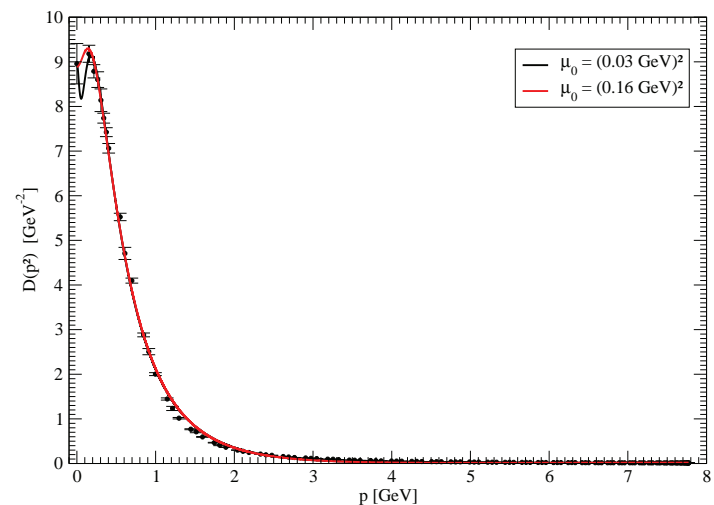

(b) Reconstructed propagator.

Figure 3: Results for the gluon spectral function and the reconstructed propagator vs. the input data. We refer to the main text and [16] for additional details.

the gluon spectral density is indeed a nonpositive quantity. One can also compare our estimate for the gluon spectral function, based on lattice data, with the numerical output of solving the complex momentum Dyson-Schwinger equations. With our current results, we do not see evidence of the reported sharp peak of [20], while the violation of positivity sets in well before $\omega \sim 600 \mathrm{MeV}$ [20].

\section{Gluons at finite temperature}

In this section, we consider lattice results for the gluon propagator at finite temperature. We study positivity violation through the computation of the Schwinger and spectral functions, and also consider the interpretation of the gluon propagator as a massive propagator.

The lattice setup for the simulations at finite temperature considered here is described in Table 1. For further details see [17].

\subsection{Positivity violation and spectral densities}

In this subsection, the temporal correlator defined in eq. (1.2) is computed for the longitudinal and transverse components of the gluon propagator for the temperatures described in Table 1. The
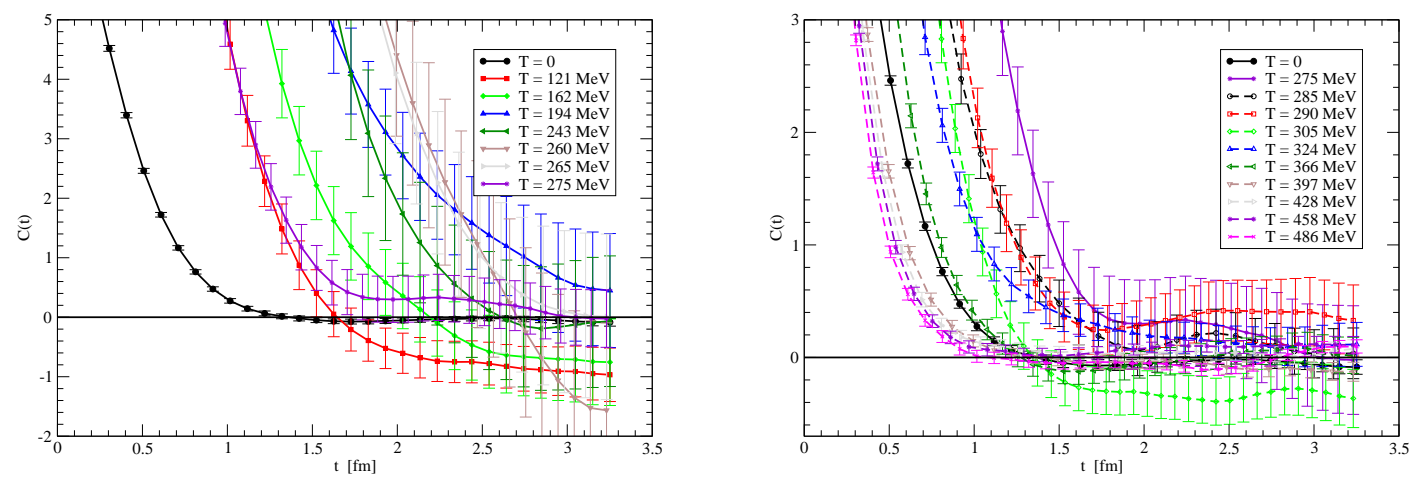

Figure 4: Temporal correlator for the longitudinal component. 


\begin{tabular}{cccclc}
\hline $\begin{array}{c}\text { Temp. } \\
(\mathrm{MeV})\end{array}$ & $\beta$ & $L_{s}$ & $L_{t}$ & $\begin{array}{c}a \\
(\mathrm{fm})\end{array}$ & $\begin{array}{c}1 / a \\
(\mathrm{GeV})\end{array}$ \\
\hline 121 & 6.0000 & 64 & 16 & 0.1016 & 1.9426 \\
162 & 6.0000 & 64 & 12 & 0.1016 & 1.9426 \\
194 & 6.0000 & 64 & 10 & 0.1016 & 1.9426 \\
243 & 6.0000 & 64 & 8 & 0.1016 & 1.9426 \\
260 & 6.0347 & 68 & 8 & 0.09502 & 2.0767 \\
265 & 5.8876 & 52 & 6 & 0.1243 & 1.5881 \\
275 & 6.0684 & 72 & 8 & 0.08974 & 2.1989 \\
285 & 5.9266 & 56 & 6 & 0.1154 & 1.7103 \\
290 & 6.1009 & 76 & 8 & 0.08502 & 2.3211 \\
305 & 6.1326 & 80 & 8 & 0.08077 & 2.4432 \\
324 & 6.0000 & 64 & 6 & 0.1016 & 1.9426 \\
366 & 6.0684 & 72 & 6 & 0.08974 & 2.1989 \\
397 & 5.8876 & 52 & 4 & 0.1243 & 1.5881 \\
428 & 5.9266 & 56 & 4 & 0.1154 & 1.7103 \\
458 & 5.9640 & 60 & 4 & 0.1077 & 1.8324 \\
486 & 6.0000 & 64 & 4 & 0.1016 & 1.9426 \\
\hline
\end{tabular}

Table 1: Lattice setup used for the computation of the gluon propagator at finite temperature. Simulations used the Wilson gauge action; $\beta$ was adjusted to have a constant physical volume, $L_{s} a \simeq 6.5 \mathrm{fm}$. For the generation of gauge configurations and Landau gauge fixing, we used Chroma [18] and PFFT [19] libraries.
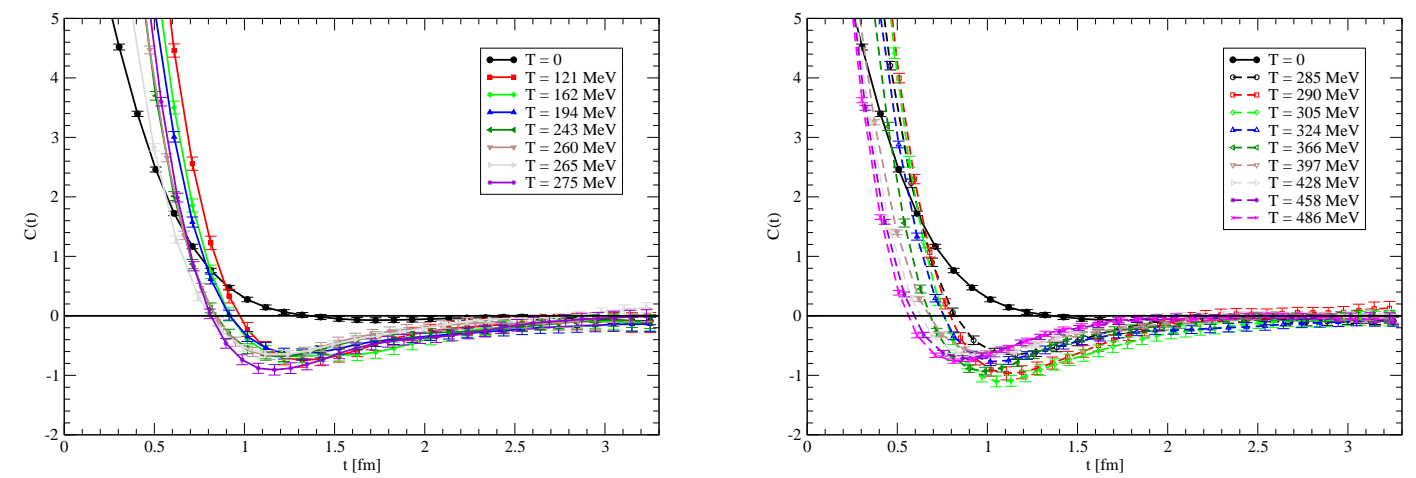

Figure 5: Temporal correlator for the transverse component.

Schwinger function for the longitudinal form factor, see Figure 4, and for the transverse form factor, see Figure 5, show that positivity is violated for both transverse and longitudinal components at all temperatures. From the results for the transverse propagator, we see that the time scale for positivity violation decreases with the temperature - see Figure 6. This suggests that, for sufficiently high temperatures, transverse gluons can behave as quasi-particles. In what concerns the behaviour of the time scale associated with the violation of positivity for the longitudinal propagator with the temperature, the results are not so clear as for the transverse Schwinger function. 


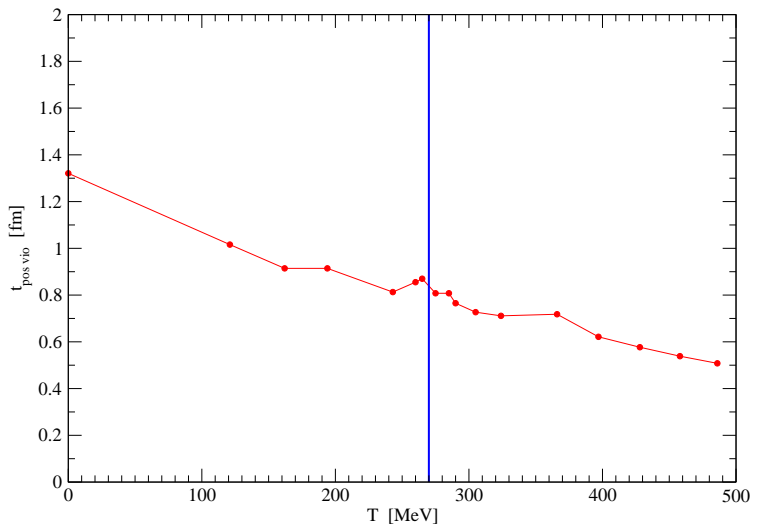

Figure 6: Positivity violation scale for the transverse propagator.
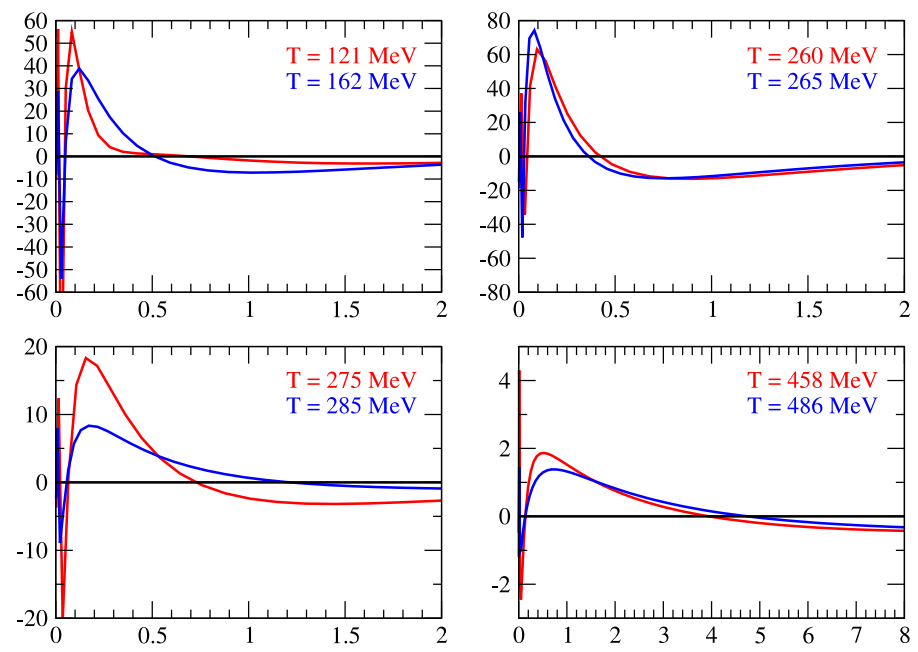

Figure 7: Longitudinal propagator spectral densities.

The spectral densities ${ }^{2}$ associated with the longitudinal gluon form factor can be seen in Figure 7. The spectral density $\rho(\mu)$ is negative for large $\mu$ and the energy scale at which the $\rho(\mu)$ becomes negative seems to increase with temperature. This, again, suggests that for sufficiently high temperatures, longitudinal gluons may be considered as massive quasi-particles.

\subsection{Gluon mass}

One can associate with the gluon propagator a mass scale. Here we consider different definitions for an electric (longitudinal) and magnetic (transverse) gluon mass scale as a function of the temperature - see [17] for details.

\footnotetext{
${ }^{2}$ For another recent work concerning gluon spectral functions at finite temperature see [21].
} 


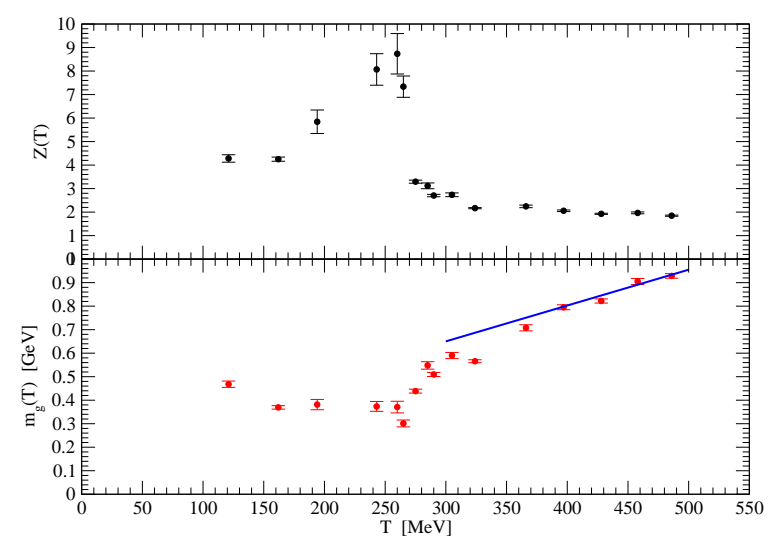

Figure 8: $Z(T)$ and $m_{g}(T)$ from fitting the longitudinal gluon propagator to a Yukawa form. The curve in the lower plot is the fit of $m_{g}$ to the functional form predicted by perturbation theory.

In a quasi-particle picture, the gluon is considered a massive boson with a Yukawa-type propagator

$$
D(p)=\frac{Z}{p^{2}+m^{2}},
$$

where $m$ is the gluon mass and $Z^{\frac{1}{2}}$ the overlap between the gluon state and the quasi-particle massive state.

A value for the gluon mass can be obtained fitting the infrared lattice data to eq. (2.1). In Table 2 and in Figure 8 we report the functions $Z(T)$ and $m_{g}(T)$ associated with the longitudinal component of the propagator. In what concerns the transverse form factor, it turns out that it is not described by a Yukawa-like function in the infrared region and one concludes that the magnetic propagator does not behave as a quasi-particle massive boson for $T \lesssim 500 \mathrm{MeV}$.

Another nonperturbative mass scale associated with gluon propagator that can be defined is given by

$$
m=1 / \sqrt{D\left(p^{2}=0 ; T\right)} .
$$

Such a mass scale is reported in Figure 9 for both electric and magnetic components. Moreover, in the right-hand plot we compare our data for the electric component with the results given in [22] using the same renormalization condition.

\section{Acknowledgments}

Work supported by FCT via projects CERN/FP/123612/2011, CERN/FP/123620/2011, and PTDC/FIS/100968/2008, developed under the initiative QREN financed by the UE/FEDER through the Programme COMPETE - Programa Operacional Factores de Competitividade. P. J. Silva supported by FCT grant SFRH/BPD/40998/2007. D. Dudal acknowledges financial support from the Research-Foundation Flanders (FWO Vlaanderen) via the Odysseus grant of F. Verstraete. 


\begin{tabular}{cllll}
\hline $\begin{array}{c}\text { Temp. } \\
(\mathrm{MeV})\end{array}$ & $\begin{array}{l}p_{\max } \\
(\mathrm{GeV})\end{array}$ & $Z(T)$ & $\begin{array}{l}m_{g}(T) \\
(\mathrm{GeV})\end{array}$ & $\chi^{2} /$ d.o.f. \\
\hline 121 & 0.467 & $4.28(16)$ & $0.468(13)$ & 1.91 \\
162 & 0.570 & $4.252(89)$ & $0.3695(73)$ & 1.66 \\
194 & 0.330 & $5.84(50)$ & $0.381(22)$ & 0.72 \\
243 & 0.330 & $8.07(67)$ & $0.374(21)$ & 0.27 \\
260 & 0.271 & $8.73(86)$ & $0.371(25)$ & 0.03 \\
265 & 0.332 & $7.34(45)$ & $0.301(14)$ & 1.03 \\
275 & 0.635 & $3.294(65)$ & $0.4386(83)$ & 1.64 \\
285 & 0.542 & $3.12(12)$ & $0.548(16)$ & 0.76 \\
290 & 0.690 & $2.705(50)$ & $0.5095(85)$ & 1.40 \\
305 & 0.606 & $2.737(80)$ & $0.5900(32)$ & 1.30 \\
324 & 0.870 & $2.168(24)$ & $0.5656(63)$ & 1.36 \\
366 & 0.716 & $2.242(55)$ & $0.708(13)$ & 1.80 \\
397 & 0.896 & $2.058(34)$ & $0.795(11)$ & 1.03 \\
428 & 1.112 & $1.927(24)$ & $0.8220(89)$ & 1.30 \\
458 & 0.935 & $1.967(37)$ & $0.905(13)$ & 1.45 \\
486 & 1.214 & $1.847(24)$ & $0.9285(97)$ & 1.55 \\
\hline
\end{tabular}

Table 2: Results of fitting the longitudinal propagator $D_{L}\left(p^{2}\right)$ to a Yukawa form from $p=0$ up to $p_{\max }$.

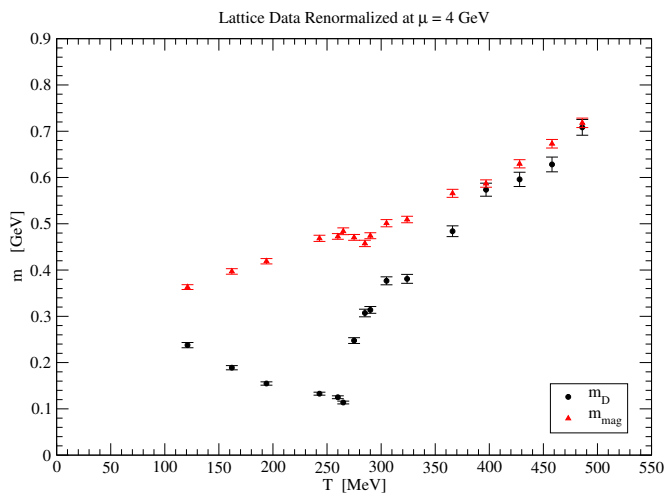

(a) Our results.

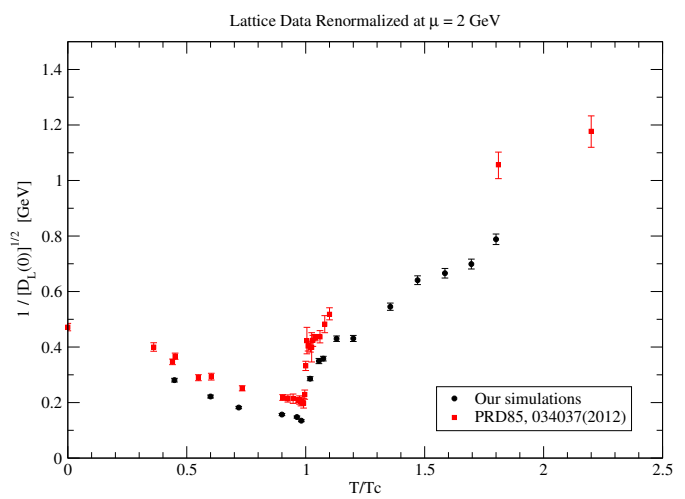

(b) Comparison with [22].

Figure 9: Electric and magnetic mass defined from zero momentum propagators.

\section{References}

[1] A. Cucchieri and T. Mendes, PoS (LATtICE 2007) 297.

[2] I. L. Bogolubsky, E. M. Ilgenfritz, M. Muller-Preussker and A. Sternbeck, Phys. Lett. B 676 (2009) 69.

[3] O. Oliveira and P. J. Silva, Phys. Rev. D 86 (2012) 114513.

[4] P. J. Silva, O. Oliveira, Nucl. Phys. B 690 (2004) 177-198. 
[5] A. Sternbeck, M. Müller-Preussker, Phys. Lett. B 726 (2013) 396-403.

[6] V. G. Bornyakov, E.-M. Ilgenfritz, C. Litwinski, V. K. Mitrjushkin, M. Müller-Preussker, arXiv:1302.5943 [hep-lat].

[7] A. Cucchieri, T. Mendes and A. R. Taurines, Phys. Rev. D 71 (2005) 051902.

[8] C. Aubin and M. C. Ogilvie, Phys. Rev. D 70 (2004) 074514.

[9] P. J. Silva and O. Oliveira, PoS (LAT2006) 075.

[10] P. O. Bowman, U. M. Heller, D. B. Leinweber, M. B. Parappilly, A. Sternbeck, L. von Smekal, A. G. Williams and J.-b. Zhang, Phys. Rev. D 76 (2007) 094505.

[11] J. M. Cornwall, arXiv:1310.7897 [hep-ph].

[12] M. Asakawa, T. Hatsuda and Y. Nakahara, Prog. Part. Nucl. Phys. 46 (2001) 459.

[13] O. Oliveira, D. Dudal and P. J. Silva, PoS (Lattice 2012) 214.

[14] D. Dudal, P. J. Silva and O. Oliveira, PoS (Confinement X) 033.

[15] P. J. Silva, D. Dudal, O. Oliveira, PoS (LATTICE 2013) 366, arXiv:1311.3643 [hep-lat].

[16] D. Dudal, O. Oliveira and P. J. Silva, Phys. Rev. D, to appear, arXiv:1310.4069 [hep-lat].

[17] P. J. Silva, O. Oliveira, P. Bicudo and N. Cardoso, arXiv:1310.5629 [hep-lat] .

[18] R. G. Edwards and B. Joó, Nucl.Phys.Proc.Suppl. 140 (2005) 832 [arXiv : hep-lat 0409003 [hep-lat] ].

[19] M. Pippig, SIAM J. Sci. Comput. 35, C213 (2013).

[20] S. Strauss, C. S. Fischer and C. Kellermann, Phys. Rev. Lett. 109 (2012) 252001.

[21] M. Haas, L. Fister, J. M. Pawlowski, arXiv:1308.4960 [hep-ph] .

[22] A. Maas, J. M. Pawlowski, L. von Smekal, and D. Spielmann, Phys. Rev. D 85 (2012) 034037. 